\title{
Mehr Remissionen mit Aripiprazol
}

Fragestellung: Erhöht Aripiprazol die Wahrscheinlichkeit einer Remission bei über 60-jährigen Patienten mit therapieresistenter Depression?

Hintergrund: Depression ist eine häufige Erkrankung bei älteren Patienten. Die Behandlung erfolgt meist durch einen Allgemeinarzt. Ein großes Problem ist die Therapieresistenz nach Einsatz von Medikamenten der ersten Wahl. Etwa 55-81\% der älteren depressiven Patienten remittieren nicht bei Behandlung mit einem selektiven Serotonin-Wiederaufnahmehemmer (SSRI) oder Serotonin-Noradrenalin-Wiederaufnahmehemmern (SNRI). Leider existieren im Gegensatz zur Behandlung von jüngeren Patienten keine Daten aus kontrollierten Studien für Medikamente der zweiten Wahl oder zur Augmentationstherapie mit Lithium, Psychostimulantien oder Antipsychotika. Ohne diese Evidenz können jedoch Nutzen und Risiken dieser Behandlung nicht abgewogen werden.

Das atypische Antipsychotikum ist bei Patienten bis 65 Jahren wirksam in der Augmentation zu SSRI oder SNRI. Dabei wurden neurologische und kardiometabolische unerwünschte

Lenze EJ, Mulsant BH, Blumberger DM et al. Efficacy, safety, and tolerability of augmentation pharmacotherapy with aripiprazole for treatment-resistant depression in late life: a randomised, double-blind, placebocontrolled trial. Lancet 2015; 386: 2404- 12
Arzneiwirkungen wie Akathisie oder Gewichtszunahme beobachtet, die bei älteren Patienten erst recht auftreten könnten. Nicht zuletzt sind Antipsychotika bei älteren Patienten mit Demenz mit erhöhter Mortalität assoziiert, die möglicherweise durch Verlängerung der QTc-
Zeit, Arrythmien oder plötzlichen Herztod verursacht wird. Die Augmentation mit Aripiprazol könnte einen Nutzen, aber auch ein Risiko bei älteren Patienten mit Depression haben, was aber bislang unzureichend untersucht wurde.

Patienten und Methodik: Die randomisierte, placebokontrollierte Doppelblindstudie schloss 468 Patienten aus drei Zentren in den USA und Kanada mit mindestens moderater Depression (Montgomery Åsberg Depression Rating Scale, MADRS, mindestens 15 Punkte) ein. Demenz, Psychose und Suchterkrankungen waren Ausschlusskriterien. 181 Patienten remittierten nicht unter der Standardbehandlung mit Venlafaxin (MADRS mindestens 10 in zwei aufeinanderfolgenden Untersuchungen über mindestens drei Monate). Davon wurden 91 Patienten auf Aripiprazol (Beginn mit $2 \mathrm{mg}$, Hochtitration bis $10 \mathrm{mg}$, maximal $15 \mathrm{mg}$ ) randomisiert und 90 Patienten mit Placebo weiterbehandelt. Studienziele waren Wirksamkeit und Verträglichkeit von Aripiprazol.

Ergebnisse: In der Aripiprazol-Gruppe erreichten $44 \%$ eine Remission (MADRS maximal 10 über zwei Untersuchungen), bei Placebo nur 29\% (Odds-Ratio 2,0; $95 \%$ Konfidenzintervall [KI] $1,1-3,7$; number needed to treat 6,6 ; $95 \%$-KI 3,5-81,8). Akathisie trat unter Verum in $26 \%$ der Fälle auf, unter Placebo in $12 \%$, Parkinsonismus bei $17 \%$ versus $2 \%$. Bei Suizidalität oder kardiovaskulären Ereignissen ergab sich kein Unterschied.

Schlussfolgerungen: Bei über 60-Jährigen mit therapieresistenter Depression unter Venlafaxin führt die Augmentation mit Aripiprazol zu einer wirksamen und dauerhaften Remission.

\section{- Kommentar von Markus Weih, Nürnberg}

\section{Sorgfältige Nutzen-Risiko-Abwägung erforderlich}

Eine therapieresistente Depression beim älteren Patienten ist ein sowohl häufiges als auch schweres klinisches Problem, nicht nur in den gerontopsychiatrischen Kliniken, auch in der Facharzt- oder Allgemeinarztpraxis. Gängige Kombinationen wie zum Beispiel Escitalopram oder Venlafaxin mit Lithium, Mirtazapin oder Quetiapin sind oft begrenzt durch die Multimorbidität der Patienten, geringe therapeutische Breite, aufwändiges Monitoring, Gewichtszunahme, Restless-Legs-Syndrom, kardiovaskuläre Risiken oder nicht tolerierbare Sedierung. Aripiprazol ist hier eine elegante Alternative: Seit 2004 zugelassen, hat es inzwischen in der Psychosebehandlung und bei Manien seinen festen Stellenwert und ist auch generisch erhältlich. Vom Rezeptorprofil her ist es wegen seiner partial dopaminagonistischen Wirkung her interessant. Wie bei anderen Atypika müssen auch bei Aripiprazol niedrigere Dosen als bei Psychose/Manie eingesetzt werden.

Ein Wermutstropfen sind natürlich die unerwünschten Arzneiwirkungen, vor allem die Akathisie und mehr noch, beim älteren Patienten der Parkinsonismus, die nun künftig bei schwer erkrankten Patienten gut gegen den potenziellen Nutzen abgewogen werden müssen. Vermutlich wird das Medikament ja nicht mehr in dieser Indikation offiziell zugelassen werden, aber vermutlich nach Aufklärung über den Off-LabelGebrauch ohne große Probleme bei Therapieresistenz vom Facharzt verordnet werden können.

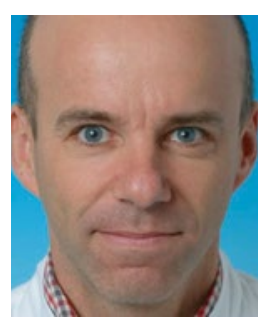

Prof. Dr. med. Markus Weih, Nürnberg

Facharzt für Neurologie, Psychiatrie

und Psychotherapie, Nervenärztliche

Gemeinschaftspraxis

E-Mail:weih@nervenaerzte-

allersbergerstrasse.de 